\title{
On Energy-Aware Dynamic Clustering for Hierarchical Sensor Networks ${ }^{\star}$
}

\author{
Joongheon Kim ${ }^{1}$, Wonjun Lee ${ }^{1, \star \star}$, Eunkyo Kim², Joonmo Kim ${ }^{3}$, \\ Choonhwa Lee ${ }^{4}$, Sungjin Kim ${ }^{1}$, and Sooyeon Kim ${ }^{5}$ \\ ${ }^{1}$ Department of Computer Science and Engineering, Korea University, Seoul, Korea \\ wlee@korea.ac.kr \\ 2 LG Electronics Institute of Technology, LG Electronics Co., Seoul, Korea \\ 3 School of Electrical, Electronics, and Computer Engineering, \\ Dankook University, Seoul, Korea \\ 4 College of Information and Communications, Hanyang University, Seoul, Korea \\ 5 Ubiquitous Computing Lab., IBM-Korea, Seoul, Korea
}

\begin{abstract}
This paper proposes an energy-efficient nonlinear programming based dynamic clustering protocol (NLP-DC) unique to sensor networks to reduce the consumption of energy of cluster heads and to prolong the sensor network lifetime. NLP-DC must cover the entire network, which is another basic functionality of topology control. To achieve these goals, NLP-DC dynamically regulates the radius of each cluster for the purpose of minimizing energy consumption of cluster heads while the entire sensor network field is still being covered by each cluster. We verify both energy-efficiency and guarantee of perfect coverage. Through simulation results, we show that NLP-DC achieves the desired properties.
\end{abstract}

\section{Introduction}

Recently many research efforts on wireless sensor networks have become one of the most active research activities in wireless communications and networks technologies [1] 2]. Sensors, the main components of wireless sensor network, are deployed over the sensing network fields, and perform the specific tasks with the processing, sensing, and communicating capacities [2]. Due to their limited power source, energy consumption has been concerned as the most critical factor when designing sensor network protocols. Facing this challenge and research issues, several approaches to prolong lifetime of the sensor networks, including clustering schemes and structured schemes with a two-tiered hierarchy, have been investigated. The clustering technology facilitates the distribution of control over the network and enables locality of communications [3]. The twotiered hierarchical structuring method is an energy-efficient scheme for wireless

\footnotetext{
* This work was sponsored by SK Telecom, Korea under Contact Number KUR0405721 to Korea University and by Grant No. R01-2005-000-10267-0 from Korea Science and Engineering Foundation in Ministry of Science and Technology.

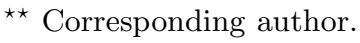


sensor networks [4]. It consists of the upper tier for communicating among cluster heads $(\mathrm{CHs})$ and the lower tier for sensing events and transmitting them to CHs. However, in traditional clustering scheme and two-tiered hierarchical structuring scheme, CHs cannot adjust their radius, which leads to inefficiency in terms of energy conservation and network management. If the cluster range is larger than optimal one, a $\mathrm{CH}$ consumes more energy than required. On the other hand, a smaller range than necessary results in the entire sensing field not being covered. To fulfill these requirements, we propose a novel clustering algorithm which aims to minimize energy consumption of $\mathrm{CHs}$ under the hierarchical structure presented in [4]. Our proposed clustering scheme, NLP-Based Dynamic Clustering (NLP-DC), is able to regulate the cluster radius for energy savings while the entire sensor network field is still being covered by each cluster. Considering the inherent features of NLP-DC, more powerful capability can be given to $\mathrm{CHs}$ than other sensor nodes. The NLP-DC could be applied to IEEE 802.15.4/ZigBee-based protocol in the aspect of energy conservation because the features of IEEE 802.15.4/ZigBee-based protocol have the different type of sensor nodes and the various type of topology (i.e., star/mesh/clustertree topology) based on clustering [5]. The remainder of this paper is organized as follows. In Section 2, we investigate previous work with focuses on clustering scheme and hierarchical structure scheme in wireless sensor networks. Section 3 describes NLP-Based Dynamic Clustering, proposed scheme in this paper. We prove the guarantee of perfect coverage of NLP-DC via theoretical analysis in Section 4. We evaluate the performance of NLP-DC via simulation in Section 5. Section 6 concludes the paper and presents the direction of our future work.

\section{Related Work}

Several clustering and hierarchical structure schemes aiming to improve energyefficiency in wireless sensor networks have been proposed. LEACH [6], a protocol architecture for sensor networks that combines the ideas of energy-efficient cluster-based routing with application-specific data aggregation to achieve good performance in terms of system lifetime, latency, and application-perceived quality. As one of the most notable clustering schemes in wireless sensor networks, it preserves limited amount of energy by selecting a $\mathrm{CH}$ at random among sensor nodes. In [7], Gupta et al. proposed a two-tiered hierarchical clustering scheme in which a $\mathrm{CH}$ with less energy constraint is selected among sensor nodes. Based on this method, it pursues an energy-efficient network management. However, a $\mathrm{CH}$ is selected among the sensor nodes as assumed in [6]. FLOC (Fast Local Clustering) [8], provides an adjustable method of cluster radius, but it is limited to one-hop or two-hop distances. The clustering-based topology control scheme [4] consists of two tiers; (1) upper tier for communicating between $\mathrm{CHs}$ and (2) lower tier for sensing, processing, and transmitting by sensors. It has similarity on load-balancing concept with NLP-DC. However, its performance depends on each radius of the cluster; as a cluster is increased covering the whole sensor network area, the energy consumption can be increased. In the previous schemes 
suggested so far, they have common features that the radius of each cluster head is fixed and mainly concentrated on sensor nodes. On the other hand, our proposed NLP-DC considers a $\mathrm{CH}$ to be a device which has more computing power and less energy-constraint. Also, it doesn't need any specific routing protocols for sensor networks since the radius of cluster radius does not concern with the distance estimated by hop count. From the next sections, we give detailed explanations on our proposed NLP-DC, which improves performance in terms of load-balanced efficient energy consumption on CHs.

\section{$3 \quad$ NLP-Based Dynamic Clustering}

The NLP-DC aims at covering the entire network with the minimum power consumptions of cluster heads by regulating the cluster range. The basic concept of NLP-DC was proposed in 9. If the cluster range is larger than optimal one, a $\mathrm{CH}$ consumes more energy than required. On the other hand, a smaller cluster range than optimal one results in the entire sensing field not being covered.

In NLP-DC, the whole sensor network field is being covered by CHs and the $\mathrm{CHs}$ consider their weights assigned by penalty functions (e.g., residual energy) and reward functions (e.g., priority). For achieving energy efficiency, a sink computes objective functions. Energy-efficient radii for each cluster are calculated based on the objective functions. Followings are what we assume in NLP-DC.

- The architecture of wireless sensor network has two-tiered hierarchical structure. The upper tier consists of $\mathrm{CHs}$ and the lower tier consists of sensors.

- A sink knows the position of each $\mathrm{CH}$.

\subsection{Initial Phase}

In initial phase, the $\mathrm{CHs}$ deployed at random construct a triangle to determine a Dynamic Clustering Controller (DCC) that is able to minimize energy consumptions of CHs while covering the entire network field as shown in Fig. 1.



Fig. 1. System model for NLP-based dynamic clustering (NLP-DC) 
The distance between DCC and each $\mathrm{CH}$ can be estimated as the radius of each cluster. Delaunay triangulation [10] [11, which guarantees the construction of an approximate equilateral triangle, is used for constructing a triangle. The construction of equilateral triangles leads to load-balanced energy consumption of each CH. The load-balanced energy consumption can prolong network lifetime.

\subsection{NLP-Based Iterative Phase}

The cluster radii of three CHs can be dynamically controlled by using a DCC as a pivot. The goal is to determine the positions of DCCs which minimize the energy consumption of each $\mathrm{CH}$ by finding energy-efficient cluster radii. As shown in Fig. 2., the triangle which is composed by three CHs. If a cluster becomes large, the $\mathrm{CH}$ of the cluster will consume more energy because the number of sensor nodes to be controlled will be increased. Therefore, if the overlapping areas of each sector are larger than optimal one, $\mathrm{CHs}$ consume more energy than required. Therefore it is important to find the minimized overlapping areas while covering the entire network. The size of overlapping area can be obtained by extracting the size of triangle from the summation of three sectors. By this concept, we can derive the objective function to find the DCC for minimizing the overlapping areas. The areas of sectors can be obtained by

$$
S=\frac{1}{2} \theta \cdot r^{2}
$$

Therefore a DCC is determined by an objective function as the Eq. (2).

$$
\begin{gathered}
\text { minimize: } f\left(r_{1}, r_{2}, r_{3}, \theta_{1}, \theta_{2}, \theta_{3}\right)=\frac{1}{2} \sum_{k=1}^{3} \theta_{k} \cdot r_{k}^{2}-S_{\text {triangle }} \\
\text { s.t. } r_{i}^{2}=\left(x_{D C C}-x_{i}\right)^{2}+\left(y_{D C C}-y_{i}\right)^{2}
\end{gathered}
$$

In Eq. (1) and Eq. (2), $\theta_{k}$ denotes the angle value of $C H_{k}, r_{k}$ means the distance between $D C C$ and $C H_{k}$. Also $S_{\text {triangle }}$ is the area of triangle which is comprised by Delaunay triangulation. As for an nonlinear programming method to solve the objective functions presented in this paper, we use an $L-B F G S$ method [12], one of the most efficient nonlinear programming methods to solve unconstraint

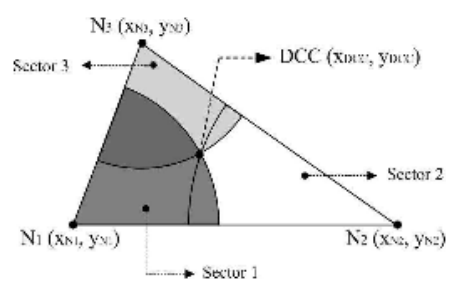

Fig. 2. Conceptual model to minimize overlapping areas 
optimization problem. As shown in the Eq. (2), the priorities in each $\mathrm{CH}$ do not considered in the objective function. However in certain cases, we need to assign a different weight to each $\mathrm{CH}$. If the $\mathrm{CH}$ in certain area is more important than the other $\mathrm{CHs}$, we need to assign higher priority to the $\mathrm{CH}$. Also if events occur in some endemic specific area, the $\mathrm{CH}$ in that area must be assigned with a higher priority. By these necessaries, the consideration of weight functions is necessary. We can consider penalty functions and reward functions for the weight functions. If the penalty function of a $\mathrm{CH}$ has a large value, the $\mathrm{CH}$ must reduce its cluster radius. If the reward function of a $\mathrm{CH}$ has a large value, the $\mathrm{CH}$ must enlarge its cluster radius. By applying this concept, we can derive the Eq. (3).

minimize: $f\left(r_{1}, r_{2}, r_{3}, \theta_{1}, \theta_{2}, \theta_{3}, \phi_{1,1}(\vec{x}), \ldots, \phi_{m, 3}(\vec{x}), \psi_{1,1}(\vec{x}), \ldots, \psi_{n, 3}(\vec{x})\right)$

$$
\begin{gathered}
=\frac{1}{2} \sum_{k=1}^{3} \theta_{k} \cdot r_{k}^{2} \cdot \prod_{l=1}^{m} \frac{\phi_{l, k}(\vec{x})}{\frac{1}{3} \sum_{l=1}^{3} \phi_{l, i}(\vec{x})} \cdot \prod_{g=1}^{n} \frac{\frac{1}{\psi_{g, k}(\vec{x})}}{\frac{1}{3} \sum_{i=1}^{3} \frac{1}{\psi_{g, i}(\vec{x})}}-S_{\text {triangle }} \\
\text { s.t. } r_{i}^{2}=\left(x_{D C C}-x_{i}\right)^{2}+\left(y_{D C C}-y_{i}\right)^{2}
\end{gathered}
$$

The notations of Eq. (3) are the same as the notations of Fig. 1, Fig. 2, Eq. (1), and Eq. (2). In Eq. (3), NLP-DC assigns a weight function to each $\mathrm{CH}$ where $\phi_{i, j}(\vec{x})$ and $\psi_{i, j}(\vec{x})$ represents a penalty function and a reward function, respectively. This objective function, Eq. (3), has $m$ penalty functions and $n$ reward functions.

\section{Guarantee of Perfect Coverage}

The guarantee of perfect coverage, the second main contribution, will be shown in this section in the aspect of theorem-based theoretical analysis.

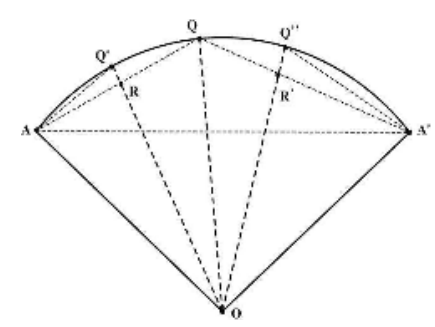

Fig. 3. Notations for corollary 1

\section{Theorem 1:}

NLP-DC can guarantee the perfect coverage. That is, the entire sensor network considered lower layer is covered by clusters in the upper layer. 


\section{Proof:}

By Corollary 1, Corollary 2, and Corollary 3, Theorem 1 can be proven.

\section{Corollary 1:}

In sector $O A A^{\prime}$ in Fig. $3, \overline{O Q^{\prime}}$, which is constructed by central point $O$ and any point, the $Q^{\prime}$, on the arc $A A^{\prime}$, meets $\overline{Q A}$ or $\overline{Q A^{\prime}}$, necessarily.

\section{Proof:}

The sector $O A A^{\prime}$ is divided into two parts as sector $O A Q$ and sector $O A^{\prime} Q$ with $Q$ (DCC in NLP-DC) considering as a reference point.

Part 1: $Q^{\prime}$ lies on arc $Q A$

When $Q^{\prime}$ lies on the $\operatorname{arc} Q A$ as shown in Fig. 4, by the property of sector $\left(\overline{O A}=\overline{O Q}=\overline{O Q^{\prime}}\right), \triangle O A Q^{\prime}$ is an isosceles triangle.

If we consider that $\angle A O Q^{\prime}=\varphi$ and $\angle A O Q=\alpha$, then $\overline{A Q^{\prime}}=$ $2 \cdot \overline{O A} \cdot \sin \frac{\varphi}{2}$. By 'the law of sines',

Then, $\overline{Q^{\prime} R}$

$$
\frac{\overline{A Q^{\prime}}}{\sin \angle A R Q^{\prime}}=\frac{\overline{Q^{\prime} R}}{\sin \angle R A Q^{\prime}}
$$

$$
\begin{aligned}
& =\frac{2 \cdot \overline{O A} \cdot \sin \frac{\varphi}{2} \cdot \sin \left(\angle O A Q^{\prime}+\angle O A Q\right)}{\sin (\angle R A O+\angle A O R)} \\
& =\frac{2 \cdot \overline{O A} \cdot \sin \frac{\varphi}{2} \cdot \sin \left(\frac{\pi-\varphi}{2}-\frac{\pi-\alpha}{2}\right)}{\sin \left(\frac{\pi-\alpha}{2}+\varphi\right)}=\frac{2 \cdot \overline{O A} \cdot \sin \frac{\varphi}{2} \cdot \sin \frac{\alpha-\varphi}{2}}{\sin \left(\frac{\pi-\alpha}{2}+\varphi\right)} .
\end{aligned}
$$

Base on the condition of $0<\varphi<\alpha<\pi$, then, $\sin \frac{\varphi}{2}>0, \sin \frac{\alpha-\varphi}{2}$ $>0$, and $\sin \left(\frac{\pi-\alpha}{2}+\varphi\right)>0$. Therefore $\overline{Q^{\prime} R}>0$. Then $\overline{O R}<\overline{O Q^{\prime}}$. Therefore the $R$ is within the sector. $R$ lies on $\overline{Q A}$ while it lies on $\overline{O Q^{\prime}}$, too. Therefore there exist a point that $\overline{O Q^{\prime}}$ meets $\overline{Q A}$.

\section{End of Proof: Part 1}

Part 2: $Q^{\prime \prime}$ lies on arc $Q A^{\prime}$

Part 2 can be proved as a same way with Part 1 . Because they are dual.

\section{End of Proof: Part 2}

\section{End of Proof: Corollary 1}

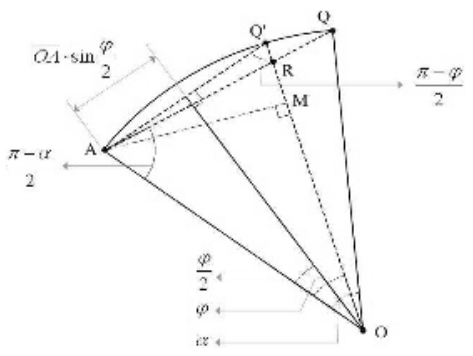

Fig. 4. Detailed description of the notations for corollary 1 


\section{Corollary 2:}

Sector $O A A^{\prime}$ in Fig. 3 covers rectangle $O A Q A^{\prime}$, perfectly.

\section{Proof:}

If we choose any $Q\left(D C C\right.$ in NLP-DC) in the arc $A A^{\prime}$,

(1) an isosceles triangle $A O Q$ is covered by a sector $A O Q$ (by Corollary 1), (2) an isosceles triangle $A^{\prime} O Q$ is covered by a sector $A^{\prime} O Q$ (by Corollary 1), (3) a sector $O A A^{\prime}=$ a sector $A O Q+$ a sector $A^{\prime} O Q$, and (4) a rectangle $O A Q A^{\prime}=$ an isosceles triangle $A O Q$ + an isosceles triangle $A^{\prime} O Q$. By (1), (2), (3), and (4), a sector $O A A^{\prime}$ covers a rectangle $O A Q A^{\prime}$, perfectly.

\section{End of Proof: Corollary 2}

\section{Corollary 3:}

The triangle constructed by Delaunay triangulation in an initial phase, is covered by three rectangles, perfectly.

\section{Proof:}

As shown in Fig. 5, when the three rectangles are united, the overlapped areas are generated while the triangle is covered, perfectly. Overlapped areas are triangle $O A_{1} B_{3}$, triangle $O A_{2} B_{1}$, and triangle $O A_{3} B_{2}$. Therefore we prove that the triangle generated in an initial phase is covered by three rectangles.

\section{End of Proof: Corollary 3}

As proved in Corollary 1, Corollary 2, and Corollary 3, rectangle $O A_{1} N_{1} B_{1}$ is covered by sector $A_{1} N_{1} B_{1}$. And rectangle $O A_{2} N_{2} B_{2}$ is covered by sector $A_{2} N_{2} B_{2}$. And rectangle $O A_{3} N_{3} B_{3}$ is covered by sector $A_{3} N_{3} B_{3}$. Also, triangle $N_{1} N_{2} N_{3}=$ rectangle $O A_{1} N_{1} B_{1}+$ rectangle $O A_{2} N_{2} B_{2}+$ rectangle $O A_{3} N_{3} B_{3}$. Then, by the triangle $N_{1} N_{2} N_{3}$, Delaunay triangle which is constructed in an initial phase, is covered by three rectangles, the triangle $N_{1} N_{2} N_{3}$ is covered by the three sectors, perfectly. That is,

$$
\text { triangle } N_{1} N_{2} N_{3} \subset \text { three rectangles } \subset \text { three sectors }
$$

Therefore, based on these three corollaries, theorem 1 is proved.

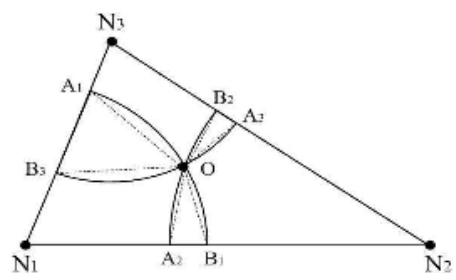

Fig. 5. Notations for corollary 3 


\section{Simulation Results}

A simulation study is conducted to evaluate the performance of NLP-DC. CHs and sensor nodes were randomly deployed. As a simulation setup, we place five CHs randomly in the upper layer. Also, performance evaluation is executed ten times. Our simulations were designed to evaluate the effect of energy-efficiency, one of the main contribution of NLP-DC. We compare NLP-DC against the method that has a fixed cluster radius, named FCR in this paper. In FCR, the $\mathrm{CHs}$ in a Delaunay triangle has cluster radii which can cover each other and be fixed. In other words, the cluster radius is fixed but it has to be extended to the distance to allow communication among neighbor $\mathrm{CHs}$ in FCR. To show the energy-efficiency, we show (1) overlapping areas and (2) residual energy.

\subsection{Overlapping Areas}

The performance of NLP-DC and FCR are compared by measuring the percentage of overlapping areas. We compared FCR and NLP-DC in hierarchical sensor network architecture. As mentioned in the head of this section, we deployed five $\mathrm{CHs}$ in upper layer and measured the percentage of overlapping areas.

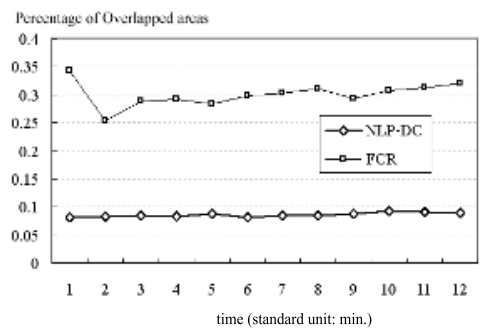

Fig. 6. The percentage of overlapping areas

When $S$ and $O A$ represents the size of network field and overlapping areas, respectively, the the percentage of overlapping areas is measured by $\frac{O A}{S}$. Fig. 6 plots the percentage of overlapping areas. As shown in Fig. 6, the percentage of overlapping areas of FCR is between 0.25 and 0.35 . On the other hands, the percentage of overlapping areas of NLP-DC is 0.08 , approximately. Therefore NLP-DC is more efficient than FCR almost from 3.125 times to 4.375 times in the aspect of the percentage of overlapping areas.

\subsection{Consumed Energy of All Cluster Heads}

The performance of NLP-DC and FCR are compared by measuring the percentage of consumed energy of CHs in this subsection.

We also compared FCR and NLP-DC in clustering-based hierarchical sensor network architecture. Fig. 7 presents the percentage of consumed energy of all 


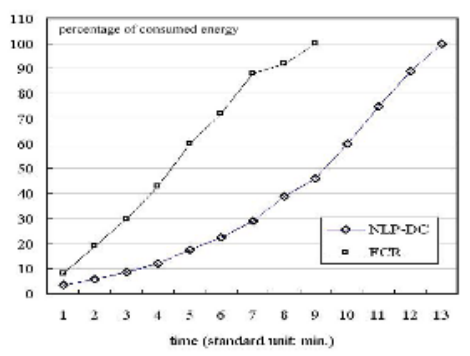

Fig. 7. The percentage of consumed energy of all cluster heads

CHs in upper layer. As shown in Fig. 7, the energy of all CHs of FCR is totally vanished between eight and nine minutes. On the other hands, the energy of all CHs of NLP-DC is vanished completely between twelve and thirteen minutes. Therefore NLP-DC is more energy-efficient than FCR almost from 1.333 times to 1.625 times in the aspect of the percentage of consumed energy of all CHs.

\section{Conclusions and Future Work}

Our proposed scheme, NLP-DC, is able to regulate the cluster radius for energy savings while the entire sensor network field is still being covered totally by each cluster in hierarchical sensor network architecture. To accomplish these objectives, NLP-DC dynamically regulates the radius of each cluster for the purpose of minimizing energy consumption of cluster heads. Therefore there exists two kinds of main contributions in this paper. We show both the 'energy-efficiency' through the design rationale of NLP-DC shown in section 3 and the 'guarantee of perfect coverage' through theorem-based theoretical analysis shown in section 4. Through simulation-based performance evaluation, the novelty on NLP-DC is shown in the aspect of energy-efficiency. More complicated situations in which sensor nodes have mobility must be considered by the emergence of wireless embedded sensors as one of dominant networking technology trends. Our future work includes the development of sensor network protocols suitable for such environments. Based on the proposed algorithm in this paper, we are developing a novel RFID reader anti-collision protocol that minimizes the overlapping areas among clusters by dynamically regulating the cluster radius among RFID readers to optimize the reader collisions. The preliminary results of the research on RFID reader anti-collision using NLP-DC is presented in [13].

\section{References}

1. D. Estrin, R. Govindan, J. Heidemann, and S. Kumar, "Next Century Challenges: Scalable Coordination in Sensor Networks," in Proc. of ACM MobiCom, Seattle, WA, USA, Aug. 1999.

2. I. F. Akyildiz, W. L. Su, Y. Sankarasubramaniam, and E. Cayirci, "Wireless Sensor Networks: A Survey", Computer Networks, Elsevier Science, 38(4), pp. 393-422, Mar. 2002. 
3. V. Mhatre and C. Rosenberg, "Design Guidelines for Wireless Sensor Networks: Communication, Clustering and Aggregation," Ad Hoc Networks Journal, Elsevier Science, 2(1), pp. 45 - 63, 2004.

4. J. Pan, Y. T. Hou, L. Cai, Y. Shi, and S. X. Shen, "Topology Control for Wireless Sensor Networks," in Proc. of ACM MobiCom, San Diego, CA, USA, Sep. 2003.

5. J. Zheng and M. J. Lee, "Will IEEE 802.15.4 Make Ubiquitous Networking a Reality?: A Discussion on a Potential Low Power, Low Bit Rate Standard," IEEE Communications Magazine, 42(6), pp. 140 - 146, Jun. 2004.

6. W. B. Heinzelman, A. P. Chandrakasan, and H. Balakrishnan, "An ApplicationSpecific Protocol Architecture for Wireless Microsensor Networks," IEEE Transactions on Wireless Communications, 1(4), pp. 660 - 670, 2002.

7. G. Gupta and M. Younis, "Load-Balanced Clustering of Wireless Sensor Networks," in Proc. of IEEE ICC, Achnorage, AK, USA, May 2003.

8. M. Demirbas, A. Arora, V. Mittal, and V. Kulathumani, "Design and Analysis of a Fast Local Clustering Service for Wireless Sensor Networks," in Proc. of IEEE BROADNETS, San Jose, CA, USA, Oct. 2004.

9. J. Kim, E. Kim, S. Kim, D. Kim, and W. Lee, "Low-Energy Localized Clustering: An Adaptive Cluster Radius Configuration Scheme for Topology Control in Wireless Sensor Networks," in Proc. of IEEE VTC, Stockholm, Sweden, May 2005.

10. M. de Berg, M. van Kreveld, M. Overmars, and O. Schwarzkopf, Computational Geometry: Algorithms and Applications, 2nd Ed., Springer-Verlag, 2000.

11. F. Aurenhammer, "Voronoi Diagrams - A Survey of a Fundamental Geometric Data Structure," ACM Computing Surveys, 23(3), pp. 345 - 405, Sep. 1991.

12. D. C. Liu and J. Nocedal, "On the Limited Memory BFGS Method for Large Scale Optimization," ACM Mathematical Programming, 45, pp. 503 - 528, Dec. 1989.

13. J. Kim, W. Lee, J. Yu, J. Myung, E. Kim, and C. Lee, "Effect of Localized Optimal Clustering for Reader Anti-Collision in RFID Networks: Fairness Aspect to the Readers," in Proc. of IEEE ICCCN, San Diego, CA, USA, Oct. 2005. 